\title{
ROLE OF PERCUTANEOUS ULTRASOUND IN CT1-3 BUCCAL MUCOSAL CARCINOMAS
}

\author{
Cecil Thankachan Thomas ${ }^{1}$, Aparna Irodi², Betty Simon ${ }^{3}$,Sramana Mukhopadhyay4, Pranay Gaikwad ${ }^{5}$
}

${ }_{1}^{1}$ Associate Professor, Department of General Surgery, Head \& Neck Oncosurgery, Christian Medical College, Vellore, Tamilnadu, India. 2Professor, Department of Radiodiagnosis, Christian Medical College, Vellore, Tamilnadu, India.

${ }_{3}^{3}$ Associate Professor, Department of Radiodiagnosis, Christian Medical College, Vellore, Tamilnadu, India.

${ }^{4}$ Associate Professor, Department of Pathology, Christian Medical College, Vellore, Tamilnadu, India.

5Professor, Department of General Surgery, Head \& Neck Oncosurgery, Christian Medical College, Vellore, Tamilnadu, India.

\begin{abstract}
BACKGROUND
The staging of buccal carcinomas has been expanded in the AJCC $8^{\text {th }}$ edition to include depth. We analysed the role of percutaneous ultrasound in the evaluation of cT1-3 buccal carcinomas. Most buccal carcinomas are commonly assessed by CT scans where the tumour may be isodense to the adjacent muscles making accurate assessment of tumour thickness difficult. There have been a few studies investigating the role of intraoral ultrasound in the assessment of thickness of oral cancers, but most of these had larger number of tongue tumours as compared to buccal lesions. We wanted to assess the correlation of thickness of buccal mucosal carcinoma by ultrasound and histopathology and its impact on the staging as per AJCC $8^{\text {th }}$ edition.
\end{abstract} ABSTRACT

\section{METHODS}

This is an observational study. A single arm of 15 patients of carcinoma buccal mucosa with no obvious skin involvement underwent percutaneous ultrasound to assess thickness and distance of tumour to skin. In this group, the overlying skin was excised if on sonographic assessment, tumour-skin distance was $<13 \mathrm{~mm}$, to achieve adequate oncological clearance while maintaining the cutaneous viability. After resection the specimen was sent for the histopathological evaluation which included assessment of depth and thickness.

\section{RESULTS}

There was positive correlation between thickness on ultrasound and histopathology (Pearson's ratio- 0.872). When depth was included to the tumour staging, there was upstaging in $26.6 \%$ of tumours and this was maintained when depth was replaced by thickness.

\section{CONCLUSIONS}

In cT1-3 buccal carcinomas, the thickness of the tumour assessed by percutaneous ultrasonography shows good correlation with histopathology and it could be used interchangeably with depth in buccal carcinomas without skin involvement.

\section{KEY WORDS}

Buccal Carcinoma, Ultrasound, Depth, Thickness, AJCC Stage

HOW TO CITE THIS ARTICLE: Thomas CT, Irodi A, Simon B, et al. Role of percutaneous ultrasound in cT1-3 buccal mucosal carcinomas. J. Evolution Med. Dent. Sci. 2019;8(26):2101-2104, DOI: 10.14260/jemds/2019/462

\section{BACKGROUND}

Oral cancer is the most common cancer affecting the Indian male and 3rd most common after cancers of the breast and uterine cervix.(1) Buccal mucosal carcinomas are common due to the widely prevalent practice of chewing betel nut with or without tobacco.(2) Many factors including the depth of invasion play a significant role in the aggressive behaviour of these lesions.(3) With the 8th edition of the AJCC, depth has become the latest additional parameter used for tumour staging.

Although tumour thickness and depth of invasion are often used synonymously, there is a theoretical difference. A

'Financial or Other Competing Interest': None.

Submission 26-04-2019, Peer Review 18-06-2019,

Acceptance 24-06-2019, Published 01-07-2019.

Corresponding Author:

Pranay Gaikwad,

Professor of Surgery and HOD,

Department of General Surgery Unit 1,

Head and Neck Oncosurgery,

Christian Medical College, Vellore-632004,

Tamilnadu, India.

E-mail: pranay@cmcvellore.ac.in

DOI: $10.14260 /$ jemds $/ 2019 / 462$ recent retrospective study concluded that the staging as per AJCC 8th edition would vary in 3.9\% patients if depth and thickness were interchangeably used.(4)

Most buccal carcinomas are commonly assessed by CT scans where the tumour may be isodense to the adjacent muscles making accurate assessment of tumour thickness difficult. There have been a few studies investigating the role of intraoral ultrasound in the assessment of thickness of oral cancers, but most of these had larger number of tongue tumours as compared to buccal lesions. Intraoral ultrasound may be inconvenient to the patient and difficult to perform when there is trismus. Percutaneous ultrasound is easy to perform, convenient and with advancement in technology, better resolution images can be obtained.

We aimed to study the accuracy of thickness of buccal carcinoma measured preoperatively on percutaneous ultrasound as compared to the histopathological thickness. We also assessed whether ultrasound can effectively guide surgical margins to achieve a negative deep margin. Further, we also sought to assess the impact of tumour thickness and depth on histopathology to the staging based on AJCC $7^{\text {th }}$ and $8^{\text {th }}$ edition and prognosis of these patients. 


\section{METHODS}

It is an observational study. A single arm study of all patients with carcinoma of the buccal mucosa attending the surgical OPD was conducted between March 2014 and June 2015. A prior Institutional Review Board (IRB) approval and institutional funding for the study was obtained. Patients who had histologically proven squamous cell carcinoma of buccal mucosa with tumour entirely in buccal mucosa or with epicenter in buccal mucosa were included. Only lesions without clinical skin involvement, in the form of ulceration and peau d'orange, were included (cT1-3 as per AJCC $7^{\text {th }}$ edition).

If the above conditions were satisfied, the patient was explained the details of the study and was recruited after a written consent was obtained. The preoperative investigations were directed towards staging and assessment of fitness for surgery. Clinical staging was done by direct examination of the lesion and cross-sectional imaging if the tumour was close to bone or retromolar trigone or if doubtful nodes were present. Chest $\mathrm{X}$ ray was done to rule out lung secondaries.

Ultrasound was performed on a Toshiba Xario ultrasound machine with a high frequency (10-15 MHz) probe. Percutaneous ultrasound was performed by placing the ultrasound probe on the cheek of the patient and scanning the whole cheek longitudinally as well transversely. Maximum tumour thickness was measured using the measurement tool callipers on the machine. Similarly, the distance from the deepest portion of the tumour to the overlying skin was also measured (Fig. 1). If it was difficult to visualise the mass, examiner would place a finger intra-orally on the tumour aiding in localising the lesion. If the distance between the deep margin of the tumour and overlying skin was less than $13 \mathrm{~mm}$, then the skin was excised along with the tumour, in order to attain a $10 \mathrm{~mm}$ surgical margin. The value of $13 \mathrm{~mm}$ was obtained by assuming that the surgical margin should be $10 \mathrm{~mm}$ and that at least $3 \mathrm{~mm}$ of subcutaneous tissue was to be kept for preserving the viability of the overlying skin. The circumferential surgical resection was not guided by the ultrasound and it was done by palpation of the induration around the ulcer and marking the margins with a $1-2 \mathrm{~cm}$ margin.

The histopathological specimen was oriented with markers on the mucosal aspect and on the deep aspect of the lesion and sent for routine histopathological examination. The pathologist assessed the size of primary lesion, thickness, depth of infiltration, grading, margin of resection, lymphovascular invasion, perineural invasion, bone invasion, nodal staging and extranodal spread.

\section{Statistical Analysis}

Pearson's correlation was used to compare ultrasound thickness, histopathological thickness and the distance from the skin.

\section{RESULTS \\ Demographics}

Fifteen patients with diagnosed carcinomas of the buccal mucosa fulfilling inclusion criteria presenting to the OutPatient Department (OPD) from March 2014 to June 2015 were included. The commonest prevalent habit was chewing tobacco $(15 / 15)$ followed by smoking $(4 / 15)$ and alcohol
(2/15). The commonest form of tobacco smoked was cigarette (3/4), followed by beedi $(1 / 4)$ and the commonest quid used was paan $(53.3 \%, 8 / 15)$. The mean duration of exposure to these risk factors in the study group prior to presentation was 22.8 yrs. (range, 2-60 yrs.). The mean pack year for smokers was 34 pack years (range, 1-110 pack years). The mean daily number of quids used by chewers was $7.2(\mathrm{n}=12)$.

There were $13(86.6 \%)$ patients with pT1-2 tumours and $2(13.3 \%)$ with pT3-4 tumours; and $2(14.3 \%)$ with pN+ and $12(85.7 \%)$ with pN0 status.

Correlation between Thickness of Lesion Measured by Ultrasound and Histopathological Examination

The median thickness of the lesion measured by transcutaneous ultrasound was $9 \mathrm{~mm}$ (Inter-quartile range= 6-14) and on histopathological examination was $6 \mathrm{~mm}$ (Interquartile range $=3-10)$. There was significant positive correlation between the two measurements (Fig. 2; Pearson correlation, $r=0.872, n=15, p<0.001$ ).

The overestimation of the thickness of the lesion by ultrasound was a mean of $4.2 \mathrm{~mm}$ (35\%). In 13/15 cases ultrasound estimates were more than histopathological estimates with a mean of $4.9 \mathrm{~mm}$. In 2 cases ultrasound underestimated the thickness compared to histopathology with a mean of $0.7 \mathrm{~mm}$.

\section{Correlation between Tumour-To-Skin Distance and Histopathological Deep Margin Status}

All the patients had a tumour-to-skin distance of less than 13 $\mathrm{mm}$ and so required excision of overlying skin with the buccal tumour. The median tumour-to-skin distance was $5.5 \mathrm{~mm}$ (Inter-quartile range, 3.2-7). The tumour-to-skin distance was correlated with the final histopathological deep margin status (Pearson's correlation coefficient $=0.427$ ). The closeness of the tumour to skin did not result in larger or smaller margins in the final histopathological margin.

\section{Correlation of Histopathological Depth and Thickness of the Lesion}

The depth was measured as the distance from the level of the adjacent normal epithelium to the deepest part of the invasion by the tumour, while the thickness was measured as the distance from the highest point of the tumour to the deepest point. The median depth was $4 \mathrm{~mm}$ (Inter-quartile range, $3-10 \mathrm{~mm}$ ). We looked at the relationship of the histological thickness and depth of the tumour. The Pearson's correlation was 0.982 , i.e. as the thickness increased so did the depth of the tumour.

\section{Thickness, Depth and AJCC Staging}

The staging of the tumour was done initially with AJCC 7 th edition and the data was reanalysed to look at the changes in staging, that the addition of depth of tumour brought, as per the AJCC $8^{\text {th }}$ edition. In 4/15 (26.6\%) cases, the histopathological tumour status was upstaged. In 2 cases the tumour was upstaged from T1 to T2, in 1 it was changed from $\mathrm{T} 1$ to $\mathrm{T} 3$ and in 1 from T2 to T4. There were no cases in which the lesion was down staged by the new criteria (this may be more relevant in carcinoma tongue where involvement of extrinsic muscle has been replaced with depth of tumour). The patients with T1 upstaged to T2 tumours had 
no recurrences and have been followed up for over 2 years. The patient with a $\mathrm{T} 2$ upstaged to $\mathrm{T} 4$ tumour received radiotherapy as the deep margin was $4 \mathrm{~mm}$. This patient also completed 2 years of follow up without any adverse events. The other patient with an initial T1 tumour was treated as per the older staging criteria with surgery alone and later developed a local recurrence in the follow up period. If the lesion was reclassified as per the AJCC $8^{\text {th }}$ edition it would be a pT3 lesion which would have necessitated adjuvant radiotherapy.

The staging was again analysed interchanging the histopathological depth of tumour with histopathological thickness to assess the latter's effect on the staging as per the AJCC $8^{\text {th }}$ edition. There were $4 / 15$ cases in which the pathological tumour status was upstaged with the same changes as mentioned above. Again, there were no cases in which the tumour was down-staged. Thus, the revised AJCC $8^{\text {th }}$ edition stage did not vary when pathological depth and thickness were interchangeably used.

The pathological nodal staging was compared against the pathological tumour staging, depth and thickness of the tumour. There were two patients with $\mathrm{pN}+$ disease, one with T1 and another with T4 stage as per the AJCC $7^{\text {th }}$ edition. But when analysed as per the depth categories in the AJCC $8^{\text {th }}$ edition, the nodal positivity was seen in lesions within the 5 $10 \mathrm{~mm}$ and $10-20 \mathrm{~mm}$ categories. There was no nodal positivity in patients with tumours with depth less than 5 $\mathrm{mm}$. Even when thickness was interchanged for depth, the same findings were arrived at. Thus, tumour depth had a better predictability about nodal positivity than the tumour staging of the previous edition of AJCC.

We looked at the predictability of nodal metastases with thickness evaluated on ultrasound. There were two patients with $\mathrm{pN}+$ disease, one each in the $0-5 \mathrm{~mm}$ and $>20 \mathrm{~mm}$ categories. Thus, a wide variation was seen in the ultrasound evaluation when compared to the pathological depth and thickness. All the patients were followed up for at least 2 years or till an event occurred. The mean follow-up period was 20 months. There were 3 recurrences over the study period including the one patient who was upstaged according to revised AJCC edition.

\section{DISCUSSION}

Depth of invasion is one of the important prognostic indicators in buccal carcinoma. There are no clear guidelines on the methodology to accurately assess the depth of invasion on imaging. The thickness of the tumour on the other hand, is easier to measure on imaging. However, measurements on CT scans may be limited as the tumour may often be isodense to surrounding muscle. Ultrasound and MRI are other imaging modalities which may provide better information of tumour thickness.

There was good correlation between measurements of thickness on percutaneous ultrasound and histopathology. But, in most cases ultrasound slightly over estimated the thickness. The difference between the values could be attributed to post-operative tumour shrinkage, placement in formalin, surrounding inflammation and sampling differences.

There have been many studies looking at the role of intraoral ultrasound in the assessment of thickness of oral cancers and this was the subject of two recent reviews.
Tarabichi et al, evaluated 10 studies that correlated tumour thickness in tongue carcinomas derived by ultrasound and histopathology and reported a pooled correlation coefficient of 0.95 (95\% confidence interval: 0.89-098, $\mathrm{P}<.0001$ ). Ultrasound slightly underestimated the tumour thickness on histopathology.(5) Klein Nulent analysed all subsites in their meta-analysis and showed a strong correlation between thickness assessed by ultrasound and histopathology for all sites (Pearson product correlation of 0.88 for tongue tumours, 0.90 for floor of mouth tumours and 0.80 for buccal tumours). Ultrasound overestimated thickness in most studies by about $1.1 \mathrm{~mm}$ and this was more pronounced in pT3-4 tumours. The bulk of the cases in this study were tongue carcinomas and the buccal subsite constituted $3 \%$ of overall cases.(6)
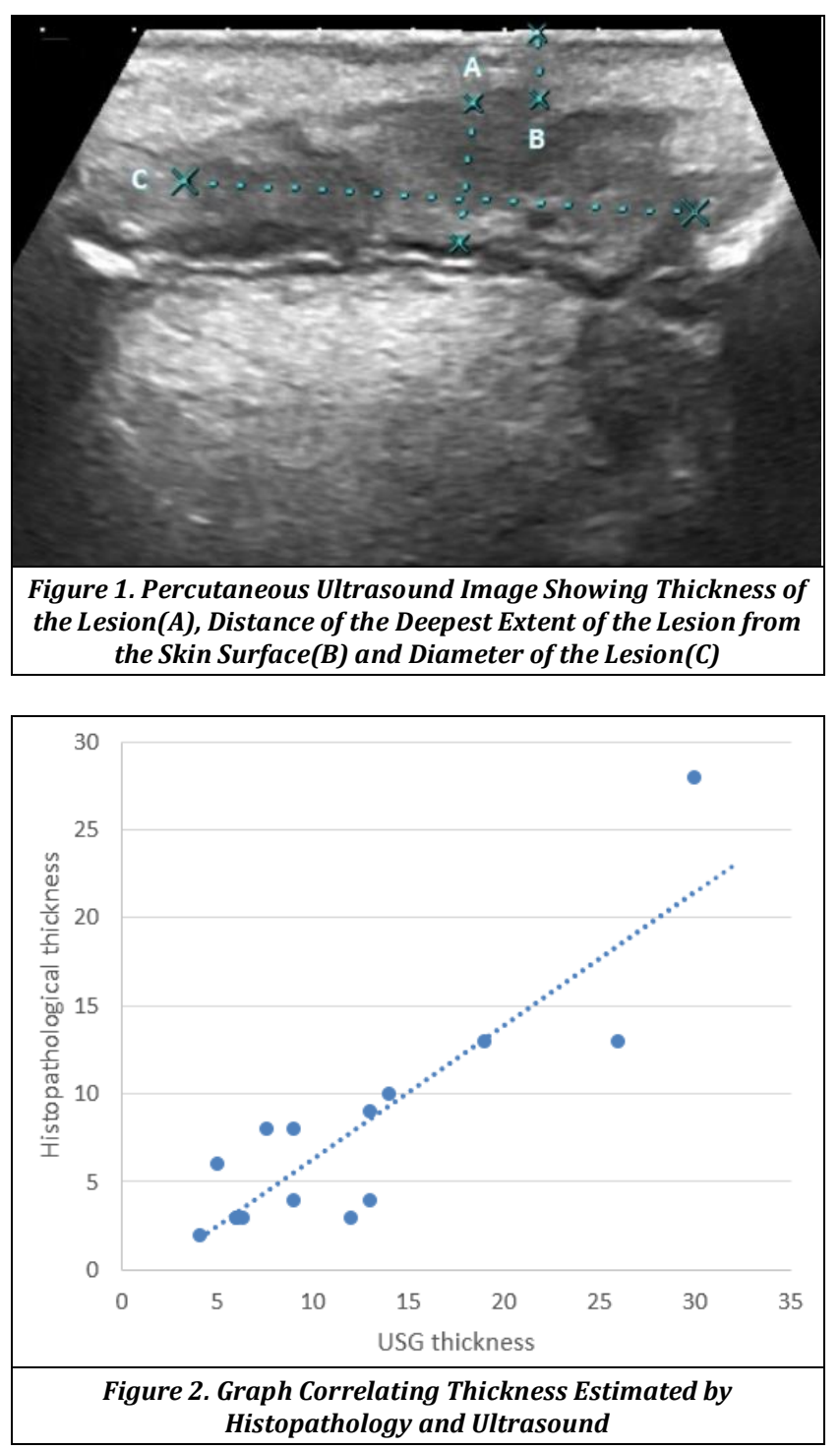

The overestimation in ultrasound can be attributed to the immediate post-resectional shrinkage of the tissues and shrinkage due to formalin fixation. Ultrasound may also pick up inflammatory changes in the deeper tissues which may add to the thickness. Mistry et al estimated the shrinkage in buccal mucosal margins after resection was $21.2 \%$.(7) Umstattd et al analysed tumour size before resection, after resection and after formalin fixation. They noted a $10.7 \%$ overall decrease in size tumour size with most of the 
decrease occurring post resectionally, before formalin fixation.(8)

Ota et al investigated the role of intraoral ultrasonography in the assessment of depth of involvement exclusively in buccal mucosal carcinoma. They proposed a classification system based on the anatomical extent into the various layers of the cheek and outlined a corresponding plan for surgical excision. When surgical treatment was performed based on their proposed classification, the disease-specific survival rate for BSCC was shown to be $73.7 \%$, with the local control rate being $89.5 \% .{ }^{(9)}$

Percutaneous ultrasound would be limited in posterior buccal lesions as there would be interference from the mandible. Otherwise, this was more comfortable to patients as compared to intraoral ultrasonography. Due to its noncontact nature of evaluation, it should theoretically distort the tumour tissue less and be more accurate.

The AJCC $8^{\text {th }}$ edition requires depth of invasion to be included in the staging. In our study, we found no variation in the revised AJCC $8^{\text {th }}$ edition stages when thickness was used as criteria instead of depth, the standard. This could be due to the smaller numbers of the study or due to the restrictive criteria including relatively early tumours without skin involvement (cT1-3, AJCC $7^{\text {th }}$ edition). In places with limited resources, ultrasound can offer a method of assessment of thickness as a surrogate to depth in clinical staging. Depth is often measured as the deepest point of the tumour from the surrounding normal epithelium. Notwithstanding varying definitions of the exact points between which this measurement is taken, there will be differences in the sampling angles due to the multiplanar orientation of a tumour. The subjectivity in assessing depth is further increased by drawing an imaginary line that corresponds to the normal level of the mucosal epithelium.(10) This is probably further complicated in imaging as the tumour tends to be seated deeper in the normal tissues by the proximity of other structures (e.g. the teeth could push a pedunculated buccal lesion deeper into the softer tissues of the buccal mucosa). Thickness has a higher objectivity to it due to the nature of the relatively fixed positions that needs to be measured, though the sampling angles can vary in performance of the ultrasound and sectioning of tumour

Nodal positivity was better predicted by depth categories than by the size criteria in T staging in our study. Mishra et al, had reported that across various T categories in 176 patients with buccal mucosal carcinomas, those tumours with a thickness greater than $4 \mathrm{~mm}$ had a higher locoregional recurrence rate than those less than $4 \mathrm{~mm}$ (51\% vs $10 \%)$.(11) Similarly, Urist et al had reported higher incidence of locoregional recurrences in buccal mucosal carcinomas with a thickness more than $6 \mathrm{~mm}$ (54\% vs. $23 \%$ ) and a depth more than $3 \mathrm{~mm}$ ( $54 \%$ vs. $26 \%$ ). On multivariate analysis for various factors including $\mathrm{T}$ stage, thickness and depth alone were significant predictors of locoregional recurrence.(12)

\section{CONCLUSIONS}

In buccal carcinomas without clinical skin involvement, the thickness of the tumour assessed by percutaneous ultrasonography shows good correlation with histopathology with ultrasound overestimating the thickness by 35\%. They can be used interchangeably with depth in buccal carcinomas without skin involvement (cT1-3). The paucity of nodal metastases in this study does not allow for any significant conclusions regarding their correlation with thickness measured by ultrasound or histopathology.

\section{REFERENCES}

[1] Ferlay J, Soerjomataram I, Ervik M, et al. GLOBOCAN 2012, v1.0, Cancer Incidence and Mortality Worldwide: IARC CancerBase No. 11, Lyon, France: International Agency for Research on Cancer: 2013. http://globocan.iarc.fr, accessed on day/month/year.

[2] KrishnaRao SV, Mejia G, Roberts-Thomson K, et al. Epidemiology of oral cancer in Asia in the past decade-an update (2000-2012). Asian Pac J Cancer Prev 2013;14(10):5567-77.

[3] Lin CS, Jen YM, Cheng MF, et al. Squamous cell carcinoma of the buccal mucosa: an aggressive cancer requiring multimodality treatment. Head Neck 2006;28(2):150-7.

[4] Dirven R, Ebrahimi A, Moeckelmann N, et al. Tumor thickness versus depth of invasion - analysis of the 8th edition American Joint Committee on Cancer Staging for oral cancer. Oral Oncol 2017;74:30-3.

[5] Tarabichi 0, Bulbul MG, Kanumuri VV, et al. Utility of intraoral ultrasound in managing oral tongue squamous cell carcinoma: systematic review. The Laryngoscope 2019;129(3):662-70.

[6] Nulent KTJW, Noorlag R, Van Cann EM, et al. Intraoral ultrasonography to measure tumor thickness of oral cancer: a systematic review and meta-analysis. Oral Oncol 2018;77:29-36.

[7] Mistry RC, Qureshi SS, Kumaran C. Post-resection mucosal margin shrinkage in oral cancer: Quantification and significance. J Surg Oncol 2005;91(2):131-3.

[8] Umstattd LA, Mills JC, Critchlow WA, et al. Shrinkage in oral squamous cell carcinoma: an analysis of tumor and margin measurements in vivo, post-resection and post-formalin fixation. Am J Otolaryngol 2017;38(6):660-2.

[9] Ota Y, Aoki T, Karakida K, et al. Determination of deep surgical margin based on anatomical architecture for local control of squamous cell carcinoma of the buccal mucosa. Oral Oncol 2009;45(7):605-9.

[10] Pentenero M, Gandolfo S, Carrozzo M. Importance of tumor thickness and depth of invasion in nodal involvement and prognosis of oral squamous cell carcinoma: a review of the literature. Head Neck 2005;27(12):1080-91.

[11] Mishra RC, Parida G, Mishra TK, et al. Tumour thickness and relationship to locoregional failure in cancer of the buccal mucosa. Eur J Surg Oncol 1999;25(2):186-9.

[12] Urist MM, O’Brien CJ, Soong SJ, et al. Squamous cell carcinoma of the buccal mucosa: analysis of prognostic factors. Am J Surg 1987;154(4):411-4. 